Article

\title{
Pseudomonas fluorescens MZ05 Enhances Resistance against Setosphaeria turcica by Mediating Benzoxazinoid Metabolism in the Maize Inbred Line Anke35
}

\author{
Cheng Zhou ${ }^{1}$, Zhongyou Ma ${ }^{1}$, Xiaoming $\mathrm{Lu}^{1}{ }^{1}$, Lin Zhu ${ }^{1, *}$ ) and Congsheng Yan ${ }^{2, *}$ \\ 1 Key Laboratory of Bio-organic Fertilizer Creation, Ministry of Agriculture, Anhui Science and Technology \\ University, Bengbu 233100, China; zhouch@ahstu.edu.cn (C.Z.); mazy@ahstu.edu.cn (Z.M.); \\ luxm@ahstu.edu.cn (X.L.) \\ 2 Institute of Horticulture, Anhui Academy of Agricultural Sciences, Hefei 230031, China \\ * Correspondence: zhul@ahstu.edu.cn (L.Z.); yancs1979@hotmail.com (C.Y.); \\ Tel.: +86-550-673-2024 (L.Z. \& C.Y.)
}

Received: 19 December 2019; Accepted: 22 January 2020; Published: 26 January 2020

check for updates

\begin{abstract}
Beneficial rhizobacteria can inhibit foliar pathogen infection by activation of defense responses, yet it the mechanisms of rhizobacteria-induced disease resistance remain largely unknown. Here, inoculation of susceptible maize plants with Pseudomonas fluorescens MZ05 significantly reduced disease occurrence caused by the leaf pathogen Setosphaeria turcica. Gene expression profiles of MZ05-inoculated plants were investigated by RNA-sequencing analyses, showing that several differentially expressed genes were positively associated with the metabolic processes of benzoxazinoids. Accordantly, the inoculation with P. fluorescens MZ05 resulted in a significant increase in the levels of 2,4-dihydroxy-7-methoxy-2H-1,4-benzoxazin-3(4H)-one (DIMBOA) in the maize leaves. Furthermore, pre-inoculation with P. fluorescens MZ05 enhanced the transcription of two defense-related marked genes $P A L$ and $P R 2 a$, as well as BX2 and GLU2, which are involved in DIMBOA biosynthesis, in pathogen-infected leaves. Defense responses in the inoculated plants were also greatly stronger and quicker than that in non-inoculated plants after pathogen attacks. However, virus-mediated silencing of BX2 or GLU2 remarkably attenuated the MZ05-induced effects, as evidenced by more disease occurrence and lower transcription of PAL and PR2a. Collectively, these findings indicated that the MZ05-induced increases of DIMBOA levels participated in the mediation of priming, which was the key mechanism in the rhizobacteria-induced host resistance.
\end{abstract}

Keywords: beneficial rhizobacteria; induced disease resistance; benzoxazinoid; Setosphaeria turcica; DIMBOA

\section{Introduction}

Plants have evolved a series of complicated defense mechanisms against pathogen infection [1,2]. Besides the physical and chemical barriers, plants possess inducible defenses that are quickly activated upon pathogen infection [3]. Numerous studies have indicated that colonization of roots by several strains of beneficial rhizobacteria can induce systemic resistance (ISR), which is effective against future pathogen invasion [4-6]. ISR is not associated with direct activation of defense responses but to a faster and stronger stimulation of basal defenses in the pathogen-infected plants $[5,6]$. In maize, the biosynthesis of defensive metabolites benzoxazinoids (BXs) can not only be induced by insects and pathogen attacks but is also generated constitutively and deposited as inactive forms in plant cells [7-9]. It has previously been shown that beneficial soil microbes can induce systemic defense by 
the BX-dependent pathways [10], indicating that the BX-mediated defense responses may be involved in the ISR systems.

Indole-3-glycerol phosphate (IGP) has been well documented to be the pivotal precursor for synthesizing Trp-independent indole-3-acetic acid (IAA) and BXs [11,12]. In some species of the grasses (Poaceae), BXs can be produced considerably during the early stages of plant growth, and gradually decline as the plant matures [13]. Many studies have revealed positive correlations between the BX levels and plant resistance $[7,14,15]$. BXs are key types of secondary metabolites widely distributed in different maize varieties or inbred lines, which possess the 2-hydroxy-2H -1,4-benzoxazin-3(4H)-one skeleton [16]. The BENZOXAZINELESS1 (BX1) gene encoding an indole-3-glycerol phosphate lyase controls the first step of the BX synthesis, which converts indole-derived IGP into free indole [13]. The BX metabolism largely depends on the levels of indole [13]. This compound can be subsequently converted by four putative cytochrome P450 monooxygenases, BX2 to BX5, into 2,4-dihydroxy-2H-1,4-benzoxazin-(4H)-one (DIBOA) [17], which can be further glucosidated by the BX8 and BX9 into DIBOA-Glc. BX6 and BX7 can catalyze the conversion of DIBOA-Glc into DIMBOA-Glc $[18,19]$. When plants experience pathogen and insect attacks, these glucosidated BXs can be rapidly hydrolyzed to the toxically active forms, such as DIBOA and DIMBOA, by $\beta$-glucosidase GLU1 or GLU2 [20]. Increasing evidence indicates that the DIMBOA is involved in defense against pathogenic fungi, aphids, and other pests, suggesting that the elevation of DIMBOA levels is an alternative defensive mechanism of plant adaptation to biotic stress $[15,16]$.

It is well demonstrated that soil microorganisms colonized in the plant rhizosphere can improve host resistance to various abiotic and biotic stresses [21,22]. These beneficial soil bacteria have generally been called plant growth-promoting rhizobacteria (PGPR), and can induce systemic resistance against herbivorous insects and foliar pathogens. Lee et al. [23] have shown that Bacillus amyloliquefaciens HK34 effectively enhance the tolerance of Panax ginseng to the foliar blight pathogen Phytophthora cactorum. Haney et al. [24] have reported that root-associated Pseudomonas sp. CH267 activates the jasmonic acid (JA)-mediated defense against the bacterial foliar pathogen Pseudomonas syringae pv. tomato DC3000. Importantly, PGPR-induced cucumber resistance against beetles has been found to be positively associated with the levels of the secondary metabolite curcubitacin [25]. More recently, rhizobacteria Bacillus spp. were shown to augment the activities of $(+)-\delta$-cadinene synthase genes and thus increase gossypol levels, which contributes to the enhanced cotton resistance against beet armyworm (Spodoptera exigua) [21]. However, it remains unclear whether PGPR can directly or indirectly drive the metabolic changes for maize defense against foliar pathogens and the underlying mechanisms of PGPR-induced host resistance.

In the present study, biochemical and molecular approaches were used to explore the mechanisms of rhizobacteria-induced maize resistance against foliar pathogens. A model experimental system consisting of susceptible maize (Zea mays) and foliar pathogen (Setosphaeria turcica) was employed to investigate the effects of the inoculation of maize plants with Pseudomonas fluorescens MZ05 on plant-pathogen interactions. Our findings provided important insights into the mechanisms underlying the process by which interactions of maize roots with P. fluorescens MZ05 activated the BX-mediated defense systems, which primarily conferred the enhanced resistance against $S$. turcica in maize plants.

\section{Materials and Methods}

\subsection{Plant and Microbial Materials}

The seeds of susceptible maize (Zea mays inbred line Anke35) were provided by Prof. Haibing Yu from the Engineering Technology Institute of Maize Breeding in Anhui Province, China. The maize inbred line Anke35 was susceptible to the hemibiotrophic fungal pathogen S. turcica (Supplementary Figure S1). Maize plants were cultured on soil and placed in a growth chamber at $25^{\circ} \mathrm{Clight}(16 \mathrm{~h}) / 22^{\circ} \mathrm{C}$ dark $(8 \mathrm{~h})$ and $80 \%$ relative humidity. Pseudomonas fluorescens strain MZ05 (GenBank No. MN611446) was isolated from rhizosphere soils of the field S. turcica-infected maize inbred line Anke35 and 
identified by $16 \mathrm{~S}$ rRNA sequencing. The fungal pathogen $S$. turcica was cultured at $26^{\circ} \mathrm{C}$ on Potato Dextrose Agar (PDA) medium before leaf inoculation.

\subsection{Assays of Microbial Inoculation}

To perform pot experiments, maize seedlings were initially grown on soil for four weeks. The isolated P. fluorescens MZ05 was cultured in liquid Luria-Bertani (LB) medium at $37^{\circ} \mathrm{C}$ in a shaker at $180 \mathrm{rpm}$ for $16 \mathrm{~h}$. The culture was centrifuged, and then washed at least three times with sterile water. Subsequently, the soil was inoculated with or without the suspensions of P. fluorescens MZ05 at the final density of $1 \times 10^{6}, 1 \times 10^{7}$, and $1 \times 10^{8} \mathrm{CFU} \mathrm{g}^{-1}$, respectively. After 7 days of soil inoculation with P. fluorescens MZ05, the collected spores of S. turcica $\left(5 \times 10^{4}\right.$ spores $\left.\mathrm{mL}^{-1}\right)$ were sprayed on the leaves of the non-inoculated and inoculated plants. After 5 days of pathogen infection, disease symptoms were monitored, and total lesion area was measured on the fourth leaves from individual plants using ImageJ software. Statistical analyses for the data were performed from three independent experiments and each experiment consisted of 15 plants.

\subsection{Analyses of DIMBOA Content by HPLC}

To quantify the content of DIMBOA, four-week-old maize seedlings were inoculated with or without bacterial suspensions of $P$. fluorescens MZ05 $\left(1 \times 10^{8} \mathrm{CFU} / \mathrm{g}\right.$ soil) for 7 days. Then, these plants were treated with $S$. turcica at $5 \times 10^{4}$ conidia $\mathrm{mL}^{-1}$ for 5 days. At the indicated time points before or after pathogen infection, the fourth leaves from 6 plants were collected and pooled to measure the content of DIMBOA, and three independent experiments were carried out. The extraction and analysis of DIMBOA by HPLC were conducted according to the method described by Song et al. [26], with minor modifications. Briefly, about $1.0 \mathrm{~g}$ of fresh maize leaves was harvested and ground in liquid nitrogen. After the addition of $5 \mathrm{~mL}$ of extraction solution (methanol/acetic acid: 49/1; v/v), these samples were sonicated for $15 \mathrm{~min}$, and then centrifuged at 12,000 g for $20 \mathrm{~min}$. The supernatants were collected to measure the content of DIMBOA by the Agilent HPLC system equipped with a reverse phase C18 column $(250 \times 4 \mathrm{~mm}, 5 \mu \mathrm{m}$ particle size $)$ and diode array detector at $280 \mathrm{~nm}$. The mobile phase was a methanol/gradient water 20:80, $5 \mathrm{~min}$; linear gradient from 20:80 to 30:70, $10 \mathrm{~min} ; 30: 70$, $20 \mathrm{~min}$; and linear gradient from 30:70 to 20:80, $30 \mathrm{~min}$. In total, $10 \mu \mathrm{L}$ of extracted samples were injected and the flow rate was kept at $1.0 \mathrm{~mL} \mathrm{~min}{ }^{-1}$. Standard DIMBOA was used to analyze the content of DIMBOA in the leaf samples.

\subsection{Development of Virus-Mediated Gene Silencing (VIGS) in Maize}

A virus-mediated gene silencing system in maize plants was developed as recently reported by Zhang et al. [27]. The VIGS vectors, including pTRV1 and pTRV2, were used for this study. Firstly, the fragment of the PDS gene encoding phytoene desaturase in maize plants was amplified by the polymerase chain reaction (PCR). The amplified product was inserted into the pTRV2 by the KpnI and BamHI digestion. Similarly, to generate the recombinant plasmids TRV2::BX2 and TRV2::GLU2, the fragments of $B X 2$ and GLU2 were amplified and inserted into pTRV2, respectively. Maize seeds were surface sterilized using $0.1 \% \mathrm{HgCl}_{2}$ for $10 \mathrm{~min}$ and then rinsed three times with sterile water. Agrobacterium tumefaciens carrying the pTRV1- and pTRV2-derived vectors (TRV2::PDS, $T R V 2:: B X 2$ or TRV2::GLU2) was cultured in LB medium at the $600 \mathrm{~nm}$ optical density value (OD600) of 0.5 , respectively. Then, the culture was mixed in a 1:1 ratio and supplemented with $20 \mathrm{mg} \mathrm{L}^{-1}$ of acetosyringone, $500 \mathrm{mg} \mathrm{L}^{-1}$ of cysteine, and $5 \mathrm{~mL} \mathrm{~L}^{-1}$ of Tween 20 . The germinated maize seeds were immersed in $50 \mathrm{~mL}$ of mixed culture, shaken, and subjected to vacuum pressure for $10 \mathrm{~min}$. The mixed culture was put into flasks and co-cultivated at $28^{\circ} \mathrm{C}$ at $180 \mathrm{rpm}$. After co-cultivation, the treated seeds were washed and cultivated in soil. The specific primers used in this experiment are shown in the Supplementary Table S1. 


\subsection{Analyses of RNA-Sequencing and Quantitative PCR ( $q P C R$ )}

For RNA-sequencing analyses, four-week-old maize seedlings were inoculated with the suspensions of $P$. fluorescens MZ05. After 7 days of inoculation, the fourth leaves of 6 non-inoculated or inoculated plants were collected and pooled to extract total RNA for the construction of cDNA libraries by the Illumina Hiseq 2500 platform (Illumina, San Diego, CA, USA). Three biological repeats were carried out for each experiment group. The Agilent 2100 Bioanalyzer was employed to detect the quality and quantity of the RNA samples. Low-quality reads and adaptor sequences were removed from raw RNA-sequencing reads, which were deposited in the NCBI SRA database (accession No. SRR10354628 and SRR10354629). The RNA-sequencing data were further analyzed as described previously by Zhou et al. [22]. Differentially expressed genes (DEGs) between the non-inoculated and inoculated plants were screened by DEGseq at FDR-adjusted $p$ value $<0.05$ and $\log _{2}$ (Fold change) $>1.0$ or $<-1.0$. Gene ontology (GO) analysis was assigned to these DEGs.

Four-week-old maize seedlings were inoculated with or without the suspensions of $P$. fluorescens MZ05 $\left(1 \times 10^{8} \mathrm{CFU} / \mathrm{g}\right.$ soil $)$ for 7 days. Then, these plants were treated with $S$. turcica at $5 \times 10^{4}$ conidia $\mathrm{mL}^{-1}$ for 5 days. At the indicated time points before or after pathogen infection, the fourth leaves from 6 plants were collected and pooled to extract total RNAs, and three independent experiments were performed. The extracted RNA samples were then reversely transcribed into cDNA using the M-MLV reverse transcriptase (Invitrogen, Carlsbad, CA, USA) according to the manufacturer's instructions. The synthesized cDNA samples were used as the templates of qPCR reactions, which were performed in an Applied Biosystems (ABI) 7500 PCR machine according to the method reported by Zhou et al. [22]. The PUBQ gene was used as the internal control for normalizing the transcription of targeted genes. The specific primers used in the $\mathrm{QPCR}$ experiments are listed in Supplementary Table S1.

\subsection{Statistical Analyses}

Statistical analyses for the data presented in this study were performed using SPSS (Chicago, IL, USA). The data indicated the means of at least three replicated experiments for each treatment. Differences among the means were compared using one-way analysis of variance (ANOVA) and Duncan's multiple range tests at $p<0.05$

\section{Results}

\subsection{P. fluorescens MZ05 Enhances Maize Resistance against S. turcica}

To investigate whether $P$. fluorescens MZ05 enhanced the resistance of maize plants against S. turcica, susceptible maize plants grown on soil were inoculated with MZ05 and subsequently subjected to pathogen infection. In this study, maize seedlings were firstly grown on soil for 4 weeks. Then, the soil was inoculated with or without the suspensions of $P$. fluorescens MZ05 at the final density of $1 \times 10^{6}$, $1 \times 10^{7}$, and $1 \times 10^{8} \mathrm{CFU} \mathrm{g}^{-1}$, respectively. After 7 days of inoculation, a spore suspension of S. turcica at $5 \times 10^{4}$ conidia $\mathrm{mL}^{-1}$ was sprayed on the leaves of both the non-inoculated (control) and inoculated plants. As shown in Figure 1a, the fourth leaves of control plants exhibited large spindle-shaped lesions surrounded by necrotic tissues at 5 days post infection (dpi), whereas the inoculation with P. fluorescens MZ05 at $1 \times 10^{6} \mathrm{CFU} / \mathrm{g}$ soil did not reduce the necrotic spots on the leaves. In contrast, plants inoculated with $P$. fluorescens MZ05 at $1 \times 10^{7}$ or $1 \times 10^{8} \mathrm{CFU} / \mathrm{g}$ soil displayed a marked reduction in the total lesion area, producing disease-resistant phenotypes (Figure 1b). Therefore, these results indicated that the higher inoculum dosage of P. fluorescens MZ05 could markedly enhance the resistance of maize plants to $S$. turcica. 
(a)

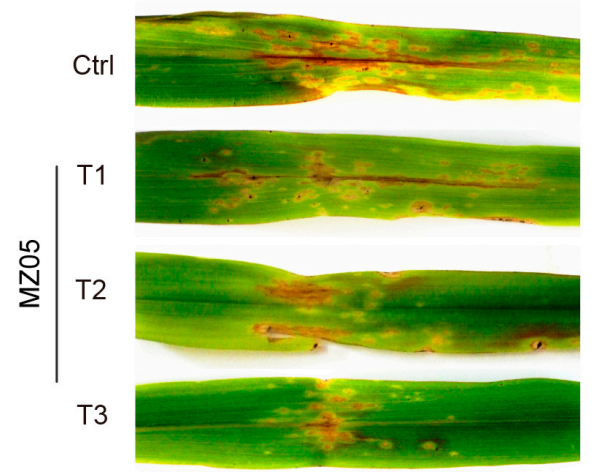

(b)

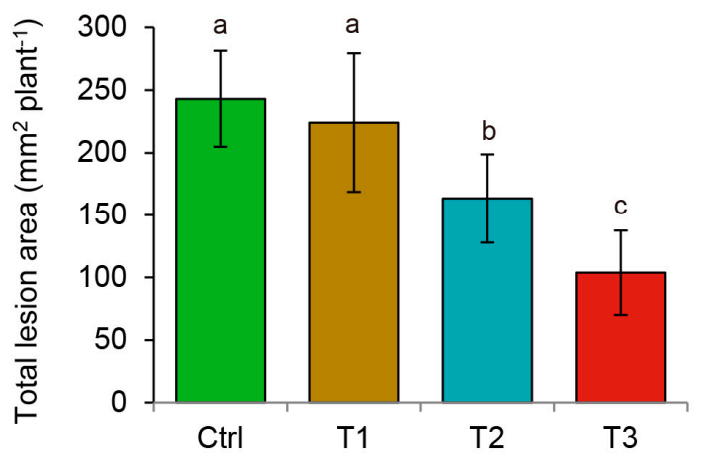

Figure 1. P. fluorescens MZ05 increased maize resistance against $S$. turcica. Four-week-old maize plants were treated with different doses $\left(\mathrm{T} 1,1 \times 10^{6} \mathrm{CFU} \mathrm{g}{ }^{-1}\right.$ soil; $\mathrm{T} 2,1 \times 10^{7} \mathrm{CFU} \mathrm{\textrm {g } ^ { - 1 }}$ soil; T3, $1 \times 10^{8} \mathrm{CFU} \mathrm{g}^{-1}$ soil) of $P$. fluorescens strain. After 7 days of inoculation, these plants were sprayed with a spore suspension of $S$. turcica at $5 \times 10^{4}$ conidia $\mathrm{mL}^{-1}$. After 5 days of pathogen infection, disease symptoms were monitored (a), and total lesion area was measured on the fourth leaves from individual plants using ImageJ software (b). Statistical analyses for the data were performed from three independent experiments and each experiment consisted of 15 plants. Different letters indicate statistically significant differences between the control (Ctrl) and inoculated plants using one-way analysis of variance and Ducan's tests $(p<0.05)$.

\subsection{Transcriptome Analyses of P. fluorescens MZ05-Inoculated Maize Plants}

To unravel the mechanisms underlying P. fluorescens MZ05's improved host disease resistance, the whole genome expression profiling of maize leaves was carried out using RNA-sequencing. Four-week-old maize plants grown on soil were inoculated with or without the presence of $P$. fluorescens MZ05 for 7 days. Then, the fourth leaves of non-inoculated (control) and inoculated plants were harvested to extract total RNAs for the analyses of RNA-sequencing. Differential gene expression was identified by the comparison of the control and inoculated plants. Compared with the control plants, 2043 genes were upregulated and 670 genes were downregulated in the inoculated plants (Supplementary Table S2). To explore the biological processes in maize plants that were regulated by P. fluorescens MZ05, gene ontology (GO) analysis was assigned to all the DEGs, and enrichment analysis was also performed by using an false discovery rate (FDR)-adjusted $p$ value $<0.05$ and fold change $>1.0$ and $<-1.0$ as the cutoff.

These DEGs were further functionally classified by the GO enrichment analysis, which were categorized into three groups: Biological process (BP), cellular component (CC), and molecular function (MF) (Figure 2a). The majority of DEGs was related to several biological pathways, such as photosynthesis, oxidation-reduction, and metabolic processes in the BP category. In the CC category, most the DEGs were significantly related to the photosystem and an integral component of membrane. In the MF category, the DEGs were involved in transferase activity, iron ion binding, oxidoreductase activity, heme binding, and transmembrane transporter activity. As mentioned above, a large number of DEGs were assigned to the metabolic process in the BP category. Among these DEGs, the transcription of two BX biosynthetic genes BX2 and GLU2 was differentially expressed in the inoculated plants compared with the controls, which was reflected by upregulation of the BX2 and GLU2 transcripts (Figure 2b). Consistently, qPCR analyses showed that the transcription levels of BX2 and GLU2 were markedly induced in maize plants after exposure to MZ05 (Figure 2c,d). In addition, qPCR was used to determine the transcription of eight randomly selected DEGs. The changing trends of these genes were in accordance with the data of RNA-sequencing (Supplementary Figure S2). 

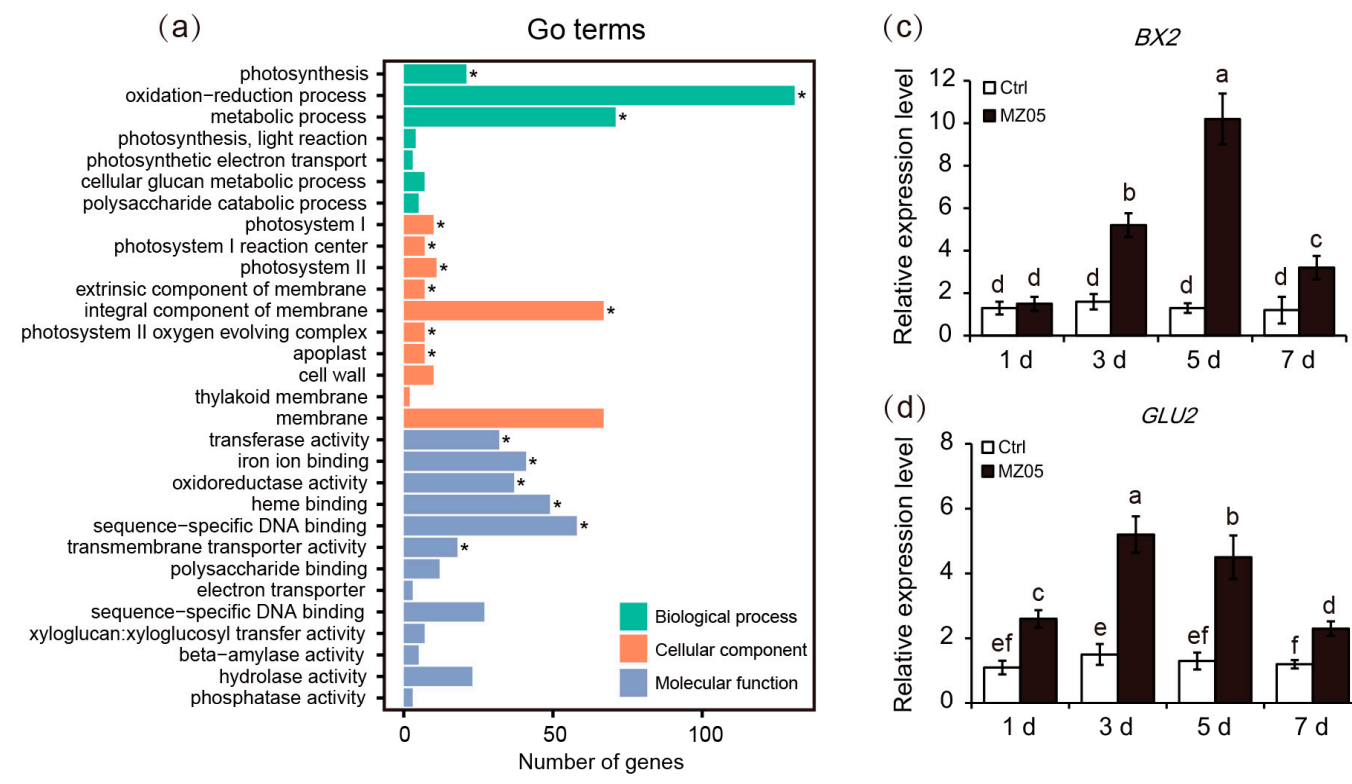

(b)

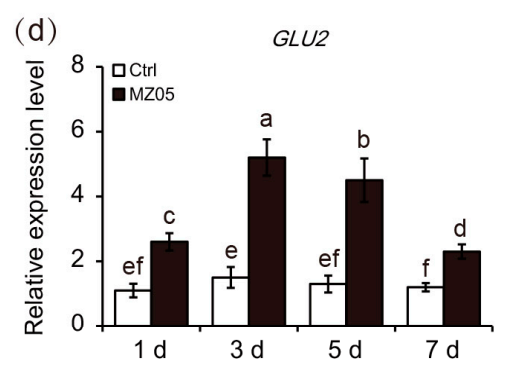

\begin{tabular}{|c|l|c|}
\hline Gene ID & \multicolumn{1}{|c|}{ Annotation } & Log $_{2}$ (Fold change) \\
\hline Zm00001d048710 & Benzoxazinone synthesis2 (BX2) & 2.9 \\
\hline Zm00001d017429 & $\begin{array}{l}\text { 4-hydroxy-7-methoxy-3-oxo-3,4-dihydro-2H-1,4- benzoxazin-2-yl glucoside beta- } \\
\text { D-glucosidase2(GLU2) }\end{array}$ & 1.7 \\
\hline
\end{tabular}

Figure 2. RNA-sequencing analyses of $P$. fluorescens MZ05-inoculated plants. Four-week-old maize seedlings were treated with or without bacterial suspensions of MZ05 $\left(1 \times 10^{8} \mathrm{CFU} / \mathrm{g}\right.$ soil $)$ for 7 days. Then, the fourth leaves were collected to extract total RNA for RNA-sequencing. (a) List of gene ontology (GO) terms for differentially expressed genes (DEGs) based on GO classifications. All of the DEGs were categorized into three groups: Biological process, cellular component, and molecular function. The asterisks indicate the significance of the comparison between the control (Ctrl) and MZ05-inoculated plants. (b) Transcription profiles of two BX biosynthetic genes BX2 and GLU2 in the inoculated plants compared with the controls. Moreover, qPCR analyses of the expression of $B X 2$ (c) and GLU2 (d) in maize plants exposed to different inoculation times of MZ05. Different letters indicate statistically significant differences between the control and inoculated plants using one-way analysis of variance and Ducan's tests $(p<0.05)$.

\subsection{Induction of Leaf DIMBOA Biosynthesis by P. fluorescens MZ05}

In order to examine if the resistance of MZ05-inoculated maize plants to $S$. turcica was positively related to the levels of DIMBOA, the content of DIMBOA was quantified in the leaves of both the control and inoculated plants. As shown in Figure 3a, no significant differences in the levels of leaf DIMBOA were observed between the control and inoculated plants after 1 day of MZ05 inoculation. However, up to 3 days after bacterial inoculation, the accumulation of DIMBOA in the MZ05-inoclauted leaves was notably higher than that in the controls (Figure 3a). The contents of DIMBOA were further increased in the leaves of plants exposed to 7 days of bacterial inoculation (Figure 3a). 
(a)
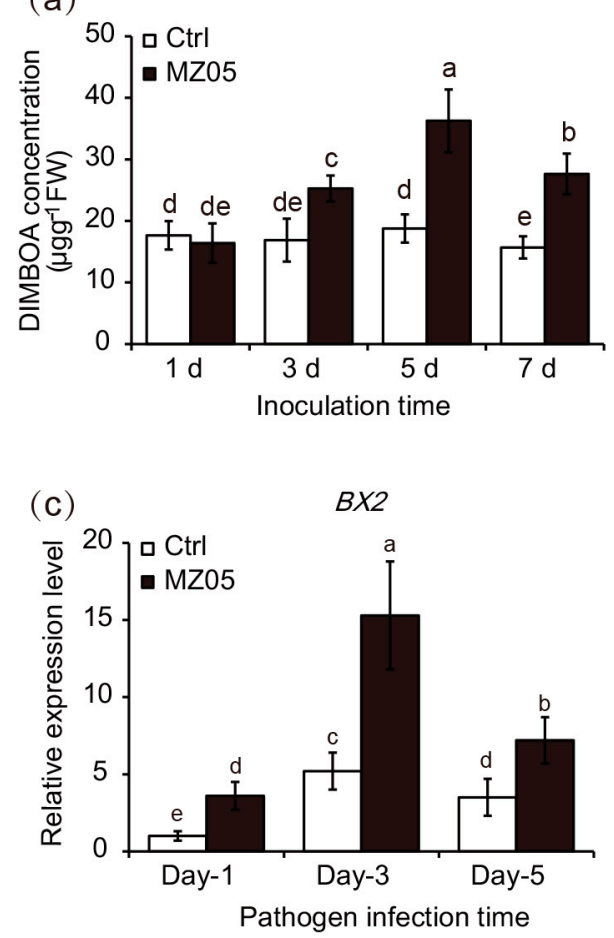

(b)

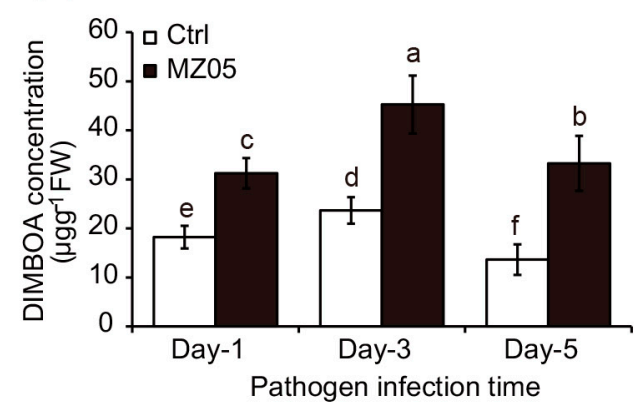

(d)

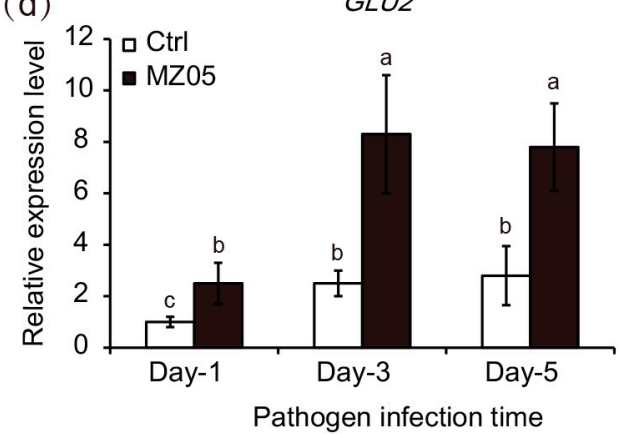

Figure 3. Analyses of the DIMBOA levels and the expression of BX biosynthetic genes in the leaves of maize plants. Four-week-old maize seedlings were treated with or without bacterial suspensions of MZ05 ( $1 \times 10^{8} \mathrm{CFU} / \mathrm{g}$ soil). The fourth leaves harvested from different inoculation times were then used to measure the levels of DIMBOA (a). After 7 days of inoculation, these plants were subjected to the infection of $S$. turcica at $5 \times 10^{4}$ conidia $\mathrm{mL}^{-1}$. At the indicated time points of pathogen infection, leaf samples were harvested to measure the content of DIMBOA (b). Meanwhile, the expression levels of BX2 (c) and GLU2 (d) were also quantified in the pathogen-infected leaves. Different letters indicate statistically significant differences between the control (Ctrl) and inoculated (MZ05) plants using one-way analysis of variance and Ducan's tests $(p<0.05)$.

The fungal pathogen S. turcica also further promoted the accumulation of DIMBOA in the maize leaves (Figure 3b). On the first day of the assays (Day-1), the levels of DIMBOA in the non-inoculated plants infected by $S$. turcica were remarkably lower than in the inoculated plants. The amount of DIMBOA was notably higher on Day- 3 and -5 than on Day- 1 in the inoculated plants and remained low in the controls with fungal infection. Furthermore, the transcription of two genes involved in the biosynthesis of DIMBOA was quantified in the pathogen-infected plants by qPCR. As shown in Figure 3c,d, the expression levels of the BX biosynthetic genes BX2 and GLU2 were markedly higher in the leaves of the inoculated plants than that of the non-inoculated plants.

\subsection{Involvement of DIMBOA in Maize Defense Responses against S. turcica}

To assess if the increased DIMBOA levels conferred the enhanced resistance of maize plants to S. turcica, we analyzed the infection degree of $S$. turcica in the leaves of empty TRV-infected control (TRV::00), TRV::BX2, and TRV::GLU2 plants with or without the presence of MZ05. In this study, a virus-mediated gene silencing system was firstly developed in the maize plants. As shown in Figure $4 \mathrm{a}$, compared to the control (TRV::00) plants, the PDS gene was largely silenced in the leaves of 2-week-old TRV::PDS plants, displaying the typical photobleaching symptoms. In addition, there were no significant differences in the phenotype and fresh weights among the control, TRV::BX2, and $T R V:: G L U 2$ plants (Figure $4 \mathrm{~b}, \mathrm{c}$ ). The $S$. turcica-infected leaves at 5 dpi were used for the observation of necrotic tissues. As shown in Figure 4d,e, the inoculation with MZ05 failed to inhibit fungal invasion and reduce lesion spots in the TRV::BX2 and TRV::GLU2 plants compared with the controls. We also 
determined the levels of DIMBOA in the leaves of pathogen-infected plants (Figure 4f). At $5 \mathrm{dpi}$, the $S$. turcica-treated control plants displayed greatly higher DIMBOA levels as compared to the $T R V:: B X 2$ or TRV::GLU2 plants. Moreover, virus-mediated silencing of BX2 or GLU2 abrogated the increased DIMBOA levels in the inoculated plants, which was consistent with the occurrence of disease symptoms.

(a)

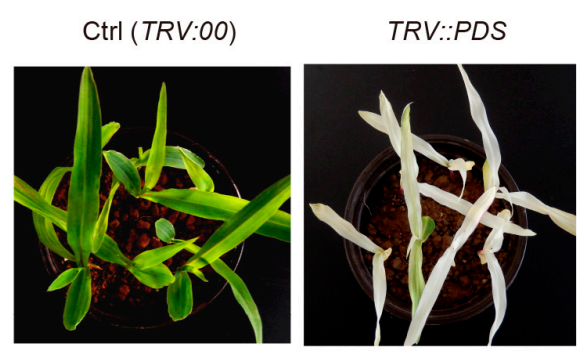

(c)

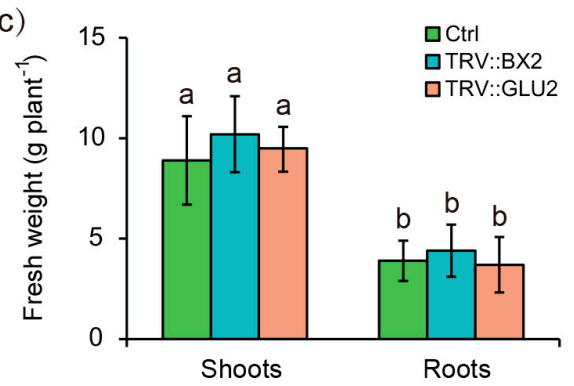

(e)

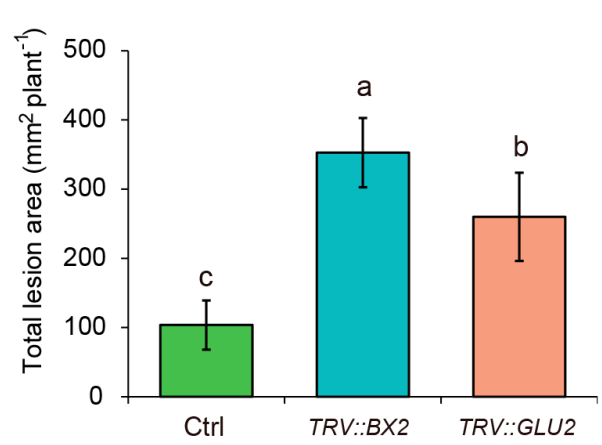

(b)

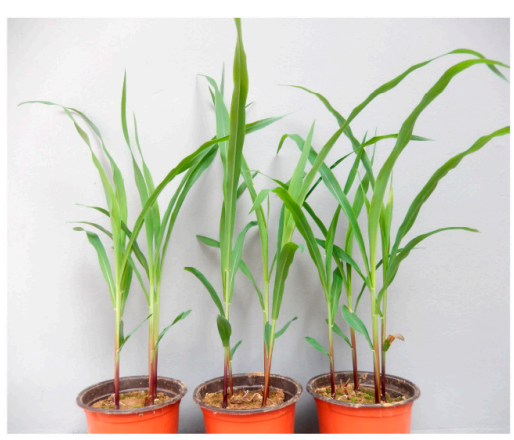

Ctrl TRV:BX2 TRV::GLU2

(d)

Ctrl

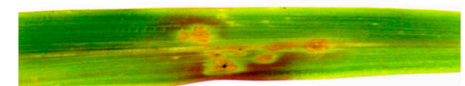

$T R V:: B \times 2$

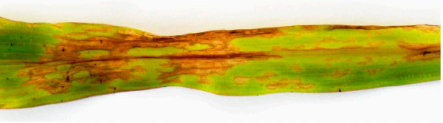

$T R V:: G L U 2$

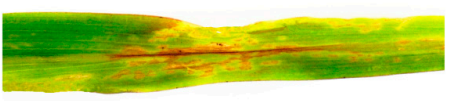

(f)

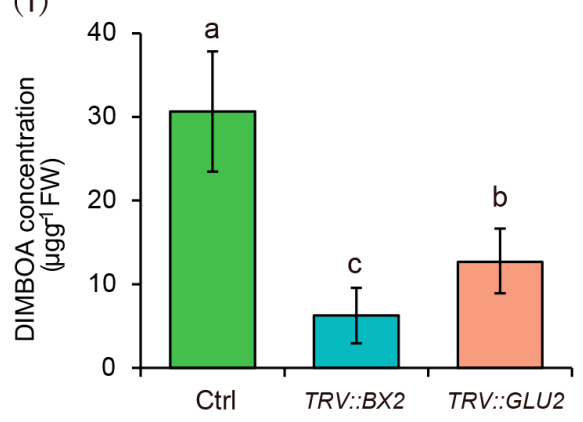

Figure 4. Virus-mediated silencing of $B X 2$ and GLU2 attenuated the P. fluorescens MZ05-induced disease resistance in maize plants. (a) The phenotype of maize plants infected with TRV::00 (Ctrl) or TRV::PDS at two weeks. (b) Maize plants were initially infected with $T R V:: 00$ (Ctrl), TRV::BX2, or TRV::GLU2 for four weeks, and (c) shoot and root fresh weights were measured in these plants. Subsequently, these plants were inoculated with P. fluorescens MZ05 $\left(1 \times 10^{8} \mathrm{CFU} / \mathrm{g}\right.$ soil) for 7 days. After that, the inoculated plants were sprayed with a spore suspension of $S$. turcica at $5 \times 10^{4}$ conidia $\mathrm{mL}^{-1}$ for 5 days. Then, these plants were used to analyze disease symptoms (d), total lesion area (e), and DIMBOA content (f). Different letters indicate statistically significant differences between the non-inoculated and inoculated plants as analyzed by Ducan's tests $(p<0.05)$.

We further examined the transcription of two defense-related marker genes, including both the phenylalanine ammonia-lyase (PAL) and $\beta$-1,3-glucanase (PR2a) genes, in the leaves of maize plants exposed to MZ05 (S) and/or S. turcica (P). The expression of $P A L$ and $P R 2 a$ was remarkably higher in response to dual inoculation $(S+P)$ than those in the non-inoculated (control) plants (Figure $5 a, b)$. By contrast, the inoculation of MZ05 or $S$. turcica alone slightly upregulated the transcription of $P A L$ and PR2a compared with the control plants (Figure $5 \mathrm{a}, \mathrm{b}$ ). However, virus-mediated silencing of $B X 2$ 
or GLU2 attenuated the MZ05-induced effects observed in pathogen-infected plants, as evidenced by lower transcription of $P A L$ and $P R 2 a$ (Figure $5 \mathrm{c}, \mathrm{d}$ ). Hence, our data indicated that the inoculation with MZ05 increased leaf DIMBOA levels and further effectively induced host defense responses, thereby hampering the pathogen infection.

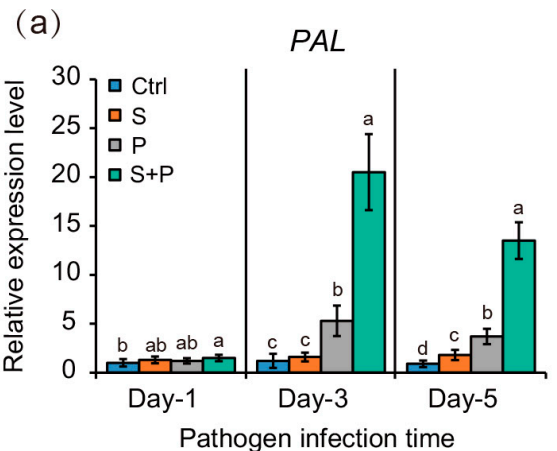

(c)

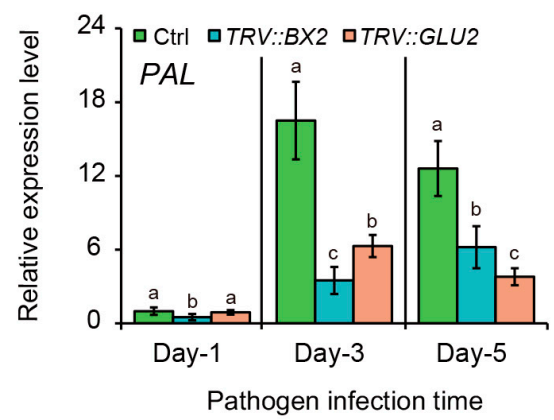

(b)

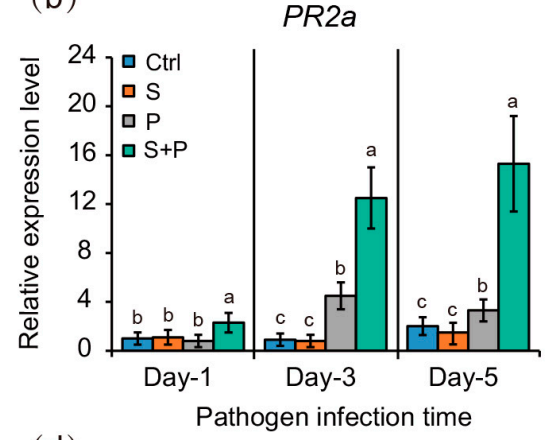

(d)

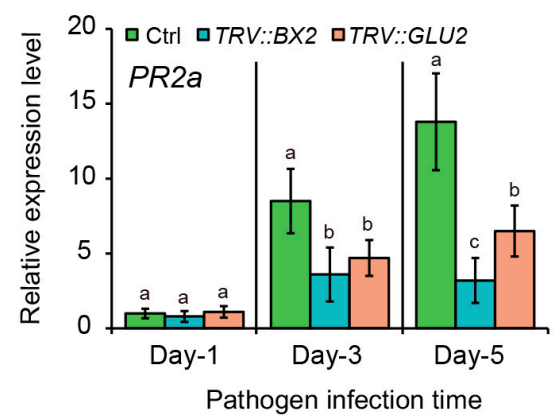

Figure 5. qPCR analyses of two defense-related marker genes $P A L$ and $P R 2 a$ in the leaves of maize plants. Four-week-old maize seedlings were inoculated with or without bacterial suspensions of MZ05 $\left(1 \times 10^{8} \mathrm{CFU} / \mathrm{g}\right.$ soil). After 7 days of inoculation, these plants were treated with the fungal pathogen $S$. turcica at $5 \times 10^{4}$ conidia $\mathrm{mL}^{-1}$. After different time points $(1,3$, and 5 days) of pathogen infection, the fourth leaves were harvested for qPCR analyses of the PAL (a) and PR2a (b) transcripts in the pathogen-infected leaves. Ctrl, control plants without pathogen and MZ05 inoculation; S: plants treated with S. turcica; P: plants inoculated with MZ05; S + P: plants inoculated with both S. turcica and MZ05. Moreover, the expression levels of $P A L(\mathbf{c})$ and $P R 2 a(\mathbf{d})$ in the pathogen-treated plants were investigated in the control (Ctrl), TRV::BX2, and TRV::GLU2 plants exposed to MZ05. Different letters indicate statistically significant differences among different groups using one-way analysis of variance and Ducan's tests $(p<0.05)$.

\section{Discussion}

The findings of the present study indicated that the beneficial soil bacterium induced the biosynthesis of BXs in the susceptible maize inbred line Anke35, with ramifications for plant-foliar pathogen interactions. Specifically, a higher accumulation of DIMBOA was detected in the leaves of $P$. fluorescens MZ05-inoculated maize plants compared with non-inoculated plants. Furthermore, the transcription levels of $B X 2$ and GLU2 involved in the biosynthesis of BXs were distinctly higher in the inoculated plants than the non-inoculated plants. The increased levels of DIMBOA in the inoculated plants were closely related to the enhanced host resistance against the necrotrophic fungus $S$. turcic. Therefore, exposure to the rhizobacteria strain P. fluorescens MZ05 can induce the biochemical and molecular alterations in maize plants, with significant ramifications for plant-pathogen interactions.

Plants can defend against multiple biotic factors by the constitutive and inducible defense mechanisms [1-3]. The inducible defenses encompass various strategies, such as the promoted biosynthesis of phytoalexins [28-31], the enhanced activities of enzymes involved in the biosynthesis of antimicrobial compounds [32-34], and the increased expression of pathogenesis-related (PR) 
proteins [35-38]. Phytoalexins have been identified as important antimicrobial metabolites for plant defense against pathogens and pests [28-31]. Its synthesis can be quickly induced by the attacks of microbial pathogens and aphids, implying that the biosynthesis of phytoalexins is one of the most pivotal defensive tactics in plants against adverse biotic factors [13,39]. BXs are one of the ubiquitous phytoalexins in gramineous plants, such as rye, wheat, and maize, and other monocotyledoneae plants that are involved in defense responses against pathogens [40-42]. DIMBOA and DIBOA are the two major types of BXs that exhibit strong inhibitory effects on microbial pathogens and pests [7]. Many studies have indicated that high levels of DIMBOA can increase plant resistance against a wide range of pathogens, such as Ostrinia nubilalis [43], Rhophalosiphum maydis [44], Diplodia maydis [45], Rhizoctonia solani [26], and Setosphaeria turcica [46]. In addition, the accumulation of DIMBOA and other hydroxamic acids in roots plays a critical role in inhibiting the growth of Gaeumannomyces graminis, the causal agent of the take-all disease of cereals, including barley and wheat [47]. In this study, the inoculation with P. fluorescens MZ05 induced a marked increase of DIMBOA levels in the susceptible maize plants, indicating that $P$. fluorescens MZ05 could be sufficient to trigger systemic synthesis of DIMBOA. We also found that pathogen infection can further promote the accumulation of DIMBOA in the inoculated leaves. It has previously been shown that DIMBOA can strongly inhibit the growth of Setosphaeria turcica in vitro [46,47]. This implied that the accumulation of leaf DIMBOA induced by MZ05 may play a vital role in the reduction of disease occurrence caused by the fungal pathogen. Similar results have been reported by a previous study, in which induction of DIMBOA synthesis by arbuscular mycorrhizal fungus (AMF) improved the disease resistance of maize plants against sheath blight caused by Rhizoctonia solani [26].

The cyclic hydroxamic acids DIBOA and DIMBOA are involved in maize defense against microbial pathogens [8-10]. In this study, the inoculation with P. fluorescens MZ05 induced increases of BX2 and GLU2, two key genes in the biosynthetic pathways of DIMBOA $[19,20]$, in the leaves of the susceptible maize inbred line Anke35. BX2 is one of the cytochrome P450-dependent monooxygenases that catalyzes consecutive hydroxylations and one ring expansion to form the highly oxidized DIBOA, which can be transformed into DIMBOA-Glc [19]. Upon exposure to insect and pathogen infection, DIBOA-Glc and DIMBOA-Glc can be further hydrolyzed by the $\beta$-glucosidases GLU1 or GLU2 to produce the bio-active BXs [20]. Previously, root colonization of AMF induces the transcription of $B X 9$ and thus leads to higher levels of DIMBOA in maize plants [26]. Therefore, transcript induction of $B X 2$ and GLU2 in the susceptible maize plants suggested induced DIMBOA biosynthesis, which was consistent with the increased levels of DIMBOA in the leaves. In the susceptible maize plants, the inoculation with P. fluorescens MZ05 significantly enhanced the transcription of BX2 and GLU2, and pathogen infection induced further increases of these genes after pathogen attack. Furthermore, P. fluorescens MZ05 did not upregulate the expression of other two defense-related genes PR2a and PAL in the leaves of maize plants, whereas pathogen infection induced high-level transcription of $P R 2 a$ and $P A L$ in leaves of the MZ05-inoculated maize plants. Upregulation of $P R 2 a$ and $P A L$ transcripts has previously been shown to effectively activate pathogen-induced plant defense responses. Here, virus-mediated silencing of $B X 2$ or GLU2 largely attenuated the enhanced accumulation of DIMBOA in the leaves of MZ05-inoculated maize plants. Furthermore, the inoculation with P. fluorescens MZ05 failed to increase the transcription of $P R 2 a$ and $P A L$, and disease resistance in both the TRV::BX2 and $T R V:: G L U 2$ plants, indicating that $P$. fluorescens MZ05 enhanced maize defense against foliar pathogen by the DIMBOA-mediated defense responses.

In fact, either necrotrophic pathogens or beneficial microbes have the ability to provoke host plants to induce priming that is a more effective defense response [48-51]. These primed plants often display stronger and quicker defense responses upon exposure to subsequent pathogen or insect attacks [49]. Ton et al. [52] have also shown that caterpillar-infested plants can release volatile organic compounds to prime defense systems for stronger and earlier induction of subsequent defense rather than to activate defense responses directly. In this study, P. fluorescens MZ05 inoculation itself did not change the transcripts of most defense genes and only upregulated the expression of two BX biosynthetic genes, 
including BX2 and GLU2. However, induction of defense responses in the pre-inoculated plants was much quicker and stronger than that in the non-inoculated plants upon pathogen infection, confirming that the BX-mediated priming effects may also be an effective defense mechanism in the P. fluorescens MZ05-induced resistance.

\section{Conclusions}

In summary, the inoculation with P. fluorescens MZ05 increased the resistance against the fungus S. turcica and caused higher levels of DIMBOA in the leaves of susceptible maize plants. In this study, a model was proposed for the MZ05-induced disease resistance, in which the inoculation with P. fluorescens MZ05 induced a significant increase of the biosynthesis gene of BXs, including BX2 and GLU2, in the plants. Upon exposure to pathogen attacks, higher accumulation of DIMBOA and upregulation of two defense-related marker genes, including $P A L$ and $P R 2 a$, were detected in the leaves of inoculated plants compared with the non-inoculated plants. Taken together, our findings indicated that the activation of BX-mediated defense responses induced by P. fluorescens MZ05 confers enhanced disease resistance in maize plants.

Supplementary Materials: The following are available online at http://www.mdpi.com/2077-0472/10/2/32/s1. Table S1: Primers used in this study. Table S2: Up-regulated and down-regulated genes differentially expressed in the P. fluorescens MZ05-inoculated plants compared with the non-inoculated plants. Figure S1: Comparative analyses of total lesion area between the susceptible (Anke35) and resistant (Anke78) maize inbred lines. Four-week-old maize seedlings were treated with S. turcica at $5 \times 10^{4}$ conidia $\mathrm{mL}^{-1}$ for $5 \mathrm{~d}$. Total lesion area was measured on the fourth leaves from individual plants using ImageJ software. Statistical analyses for the data were conducted from three independent experiments and each experiment consisted of 15 plants. Asterisk indicates significant difference between the two maize inbred lines using Student's t-test at $p<0.05$. Figure S2: qPCR analyses of eight randomly selected genes among all the DEGs.

Author Contributions: Designed experiments, L.Z. and C.Y.; writing manuscript and editing, L.Z. and C.Z.; conducted experiments, C.Z.; conducted statistical analysis, Z.M. and C.Z.; review and editing, X.L. and Z.M.; writing - original draft and review, C.Z. and L.Z.; funding acquisition, L.Z. All authors have read and agreed to the published version of the manuscript.

Funding: This research was funded by the National Natural Science Foundation of China (31600210), the China Postdoctoral Science Foundation (2017M620214), the Key Research Project of the Anhui Science and Technology Committee (16030701102), the Natural Science Foundation of the Anhui Science and Technology Committee (1908085QC110) and the Natural Science Foundation of Education Department of Anhui province (KJ2018ZD051).

Conflicts of Interest: The authors declare no conflict of interest.

\section{References}

1. Grant, M.; Lamb, C. Systemic immunity. Curr. Opin. Plant Biol. 2006, 9, 414-420. [CrossRef]

2. Ronald, P.; Joe, A. Molecular mimicry modulates plant host responses to pathogens. Ann. Bot. 2018, 121, 17-23. [CrossRef] [PubMed]

3. Bostock, R.M. Signal crosstalk and induced resistance: straddling the line between cost and benefit. Annu. Rev. Phytopathol. 2005, 43, 545-580. [CrossRef] [PubMed]

4. De Meyer, G.; Audenaert, K.; Hofte, M. Pseudomonas aeruginosa 7NSK2-induced systemic resistance in tobacco depends on in planta salicylic acid accumulation but is not associated with PR1a expression. Eur. J. Plant Pathol. 1999, 105, 513-517. [CrossRef]

5. Ryu, C.M.; Hu, C.H.; Reddy, M.S.; Kloepper, J.W. Different signaling pathways of induced resistance by rhizobacteria in Arabidopsis thaliana against two pathovars of Pseudomonas syringae. New Phytol. 2003, 160, 413-420. [CrossRef]

6. Tjamos, S.E.; Flemetakis, E.; Paplomatas, E.J.; Katinakis, P. Induction of resistance to Verticillium dahliae in Arabidopsis thaliana by the biocontrol agent K-165 and pathogenesis-related proteins gene expression. Mol. Plant Microbe Interact. 2005, 18, 555-561. [CrossRef]

7. Ahmad, S.; Veyrat, N.; Gordon-Weeks, R.; Zhang, Y.; Martin, J.; Smart, L.; Glauser, G.; Erb, M.; Flors, V.; Frey, M.; et al. Benzoxazinoid metabolites regulate innate immunity against aphids and fungi in maize. Plant Physiol. 2011, 157, 317-327. [CrossRef] 
8. Zhou, S.; Richter, A.; Jander, G. Beyond defense: multiple functions of benzoxazinoids in maize metabolism. Plant Cell Physiol. 2018, 59, 1528-1537. [CrossRef]

9. Tzin, V.; Hojo, Y.; Strickler, S.R.; Bartsch, L.J.; Archer, C.M.; Ahern, K.R.; Zhou, S.; Christensen, S.A.; Galis, I.; Mueller, L.A.; et al. Rapid defense responses in maize leaves induced by Spodoptera exigua caterpillar feeding. J. Exp. Bot. 2017, 68, 4709-4723. [CrossRef]

10. Neal, A.L.; Ton, J. Systemic defense priming by Pseudomonas putida KT2440 in maize depends on benzoxazinoid exudation from the roots. Plant Signal. Behav. 2013, 8, e22655. [CrossRef]

11. Block, A.K.; Vaughan, M.M.; Schmelz, E.A.; Christensen, S.A. Biosynthesis and function of terpenoid defense compounds in maize (Zea mays). Planta 2019, 249, 21-30. [CrossRef] [PubMed]

12. Schmelz, E.A.; Huffaker, A.; Sims, J.W.; Christensen, S.A.; Lu, X.; Okada, K.; Peters, R.J. Biosynthesis, elicitation and roles of monocot terpenoid phytoalexins. Plant J. 2014, 79, 659-678. [CrossRef] [PubMed]

13. Maschietto, V.; Lanubile, A.; Leonardis, S.D.; Marocco, A.; Paciolla, C. Constitutive expression of pathogenesis-related proteins and antioxidant enzyme activities triggers maize resistance towards Fusarium verticillioides. J. Plant Physiol. 2016, 200, 53-61. [CrossRef] [PubMed]

14. Tanaka, S.; Brefort, T.; Neidig, N.; Djamei, A.; Kahnt, J.; Vermerris, W.; Koenig, S.; Feussner, K.; Feussner, I.; Kahmann, R. A secreted Ustilago maydis effector promotes virulence by targeting anthocyanin biosynthesis in maize. eLife 2014, 3, e01355. [CrossRef]

15. Di, D.W.; Zhang, C.; Luo, P.; An, C.W.; Guo, G.Q. The biosynthesis of auxin: how many paths truly lead to IAA? Plant Growth Regul. 2016, 78, 275-285. [CrossRef]

16. Frey, M.; Stettner, C.; Pare, P.W.; Schmelz, E.A.; Tumlinson, J.H.; Gierl, A. An herbivore elicitor activates the gene for indole emission in maize. Proc. Natl. Acad. Sci. USA 2000, 97, 14801-14806. [CrossRef]

17. Kohler, A.; Maag, D.; Veyrat, N.; Glauser, G.; Wolfender, J.L.; Turlings, T.C.J.; Erb, M. Within-plant distribution of 1,4-benzoxazin-3-ones contributes to herbivore niche differentiation in maize. Plant Cell Environ. 2015, 38, 1081-1093. [CrossRef]

18. De Vleesschauwer, D.; Djavaheri, M.; Bakker, P.A.; Höfte, M. Pseudomonas fluorescens WCS374r-induced systemic resistance in rice against Magnaporthe oryzae is based on pseudobactin-mediated priming for a salicylic acid-repressible multifaceted defense response. Plant Physiol. 2008, 148, 1996-2012. [CrossRef]

19. Jonczyk, R.; Schmidt, H.; Osterrieder, A.; Fiesselmann, A.; Schullehner, K.; Haslbeck, M.; Sicker, D.; Hofmann, D.; Yalpani, N.; Simmons, C. Elucidation of the final reactions of DIMBOA-glucoside biosynthesis in maize: characterization of Bx6 and Bx7. Plant Physiol. 2008, 146, 1053-1063. [CrossRef]

20. Czjzek, M.; Cicek, M.; Zamboni, V.; Burmeister, W.P.; Bevan, D.R.; Henrissat, B.; Esen, A. Crystal structure of a monocotyledon (maize ZMGlu1) beta-glucosidase and a model of its complex with p-nitrophenyl beta-Dthioglucoside. Biochem. J. 2001, 354, 37-46. [CrossRef]

21. Zebelo, S.; Song, Y.; Kloepper, J.W.; Fadamiro, H. Rhizobacteria activates (+)- $\delta$-cadinene synthase genes and induces systemic resistance in cottonagainst beet armyworm (Spodoptera exigua). Plant Cell Environ. 2016, 39, 935-943. [CrossRef] [PubMed]

22. Zhou, C.; Zhu, L.; Guo, J.S.; Xiao, X.; Ma, Z.Y.; Wang, J.F. Bacillus subtilis STU6 ameliorates iron deficiency in tomato by enhancement of polyamine-mediated iron remobilization. J. Agric. Food Chem. 2019, 67, 320-330. [CrossRef] [PubMed]

23. Lee, B.D.; Dutta, S.; Ryu, H.; Yoo, S.J.; Suh, D.S.; Park, K. Induction of systemic resistance in Panax ginseng against Phytophthora cactorum by native Bacillus amyloliquefaciens HK34. J. Ginseng Res. 2015, 39, $213-220$. [CrossRef] [PubMed]

24. Haney, C.H.; Wiesmann, C.L.; Shapiro, L.R.; Melnyk, R.A.; O’Sullivan, L.R.; Khorasani, S.; Xiao, L.; Han, J.; Bush, J.; Carrillo, J.; et al. Rhizosphere-associated Pseudomonas induce systemic resistance to herbivores at the cost of susceptibility to bacterial pathogens. Mol. Ecol. 2018, 27, 1833-1847. [CrossRef]

25. Zehnder, G.; Yao, C.B.; Murphy, J.F.; Sikora, E.R.; Kloepper, J.W. Induction of resistance in tomato against cucumber mosaic cucumovirus by plant growth-promoting rhizobacteria. Biocontrol 2000, 45, 127-137. [CrossRef]

26. Song, Y.Y.; Cao, M.; Xie, L.J.; Liang, X.T.; Zeng, R.S.; Su, Y.J.; Huang, J.H.; Wang, R.L.; Luo, S.M. Induction of DIMBOA accumulation and systemic defense responses as a mechanism of enhanced resistance of mycorrhizal corn (Zea mays L.) to sheath blight. Mycorrhiza 2011, 21, 721-731. [CrossRef] 
27. Zhang, J.; Yu, D.; Zhang, Y.; Liu, K.; Xu, K.; Zhang, F.; Wang, J.; Tan, G.; Nie, X.; Ji, Q.; et al. Vacuum and co-cultivation agroinfiltration of (germinated) seeds results in tobacco rattle virus (TRV) mediated whole-plant virus-induced gene silencing (VIGS) in wheat and maize. Front. Plant Sci. 2017, 8, 393. [CrossRef]

28. Du, Y.; Chu, H.; Wang, M.; Chu, I.K.; Lo, C. Identification of flavone phytoalexins and a pathogen-inducible flavone synthase II gene (SbFNSII) in sorghum. J. Exp. Bot. 2010, 61, 983-994. [CrossRef]

29. Okada, A.; Okada, K.; Miyamoto, K.; Koga, J.; Shibuya, N.; Nojiri, H.; Yamane, H. OsTGAP1, a bZIP transcription factor, coordinately regulates the inductive production of diterpenoid phytoalexins in rice. J. Biol. Chem. 2009, 284, 26510-26518. [CrossRef]

30. Siciliano, I.; Amaral Carneiro, G.; Spadaro, D.; Garibaldi, A.; Gullino, M.L. Jasmonic acid, abscisic acid, and salicylic acid are involved in the phytoalexin responses of rice to Fusarium fujikuroi, a high gibberellin producer pathogen. J. Agric. Food Chem. 2015, 63, 8134-8142. [CrossRef]

31. Ube, N.; Harada, D.; Katsuyama, Y.; Osaki-Oka, K.; Tonooka, T.; Ueno, K.; Taketa, S.; Ishihara, A. Identification of phenylamide phytoalexins and characterization of inducible phenylamide metabolism in wheat. Phytochemistry 2019, 167, 112098. [CrossRef] [PubMed]

32. Narusaka, M.; Narusaka, Y. Thienopyrimidine-type compounds protect Arabidopsis plants against the hemibiotrophic fungal pathogen Colletotrichum higginsianum and bacterial pathogen Pseudomonas syringae pv. maculicola. Plant Signal. Behav. 2017, 12, e1293222. [CrossRef] [PubMed]

33. Stringlis, I.A.; de Jonge, R.; Pieterse, C.M.J. The age of coumarins in plant-microbe interactions. Plant Cell Physiol. 2019, 60, 1405-1419. [CrossRef] [PubMed]

34. Sukumaran, A.; McDowell, T.; Chen, L.; Renaud, J.; Dhaubhadel, S. Isoflavonoid-specific prenyltransferase gene family in soybean: GmPT01, a pterocarpan 2-dimethylallyltransferase involved in glyceollin biosynthesis. Plant J. 2018, 96, 966-981. [CrossRef] [PubMed]

35. Fu, Z.Q.; Dong, X. Systemic acquired resistance: turning local infection into global defense. Annu. Rev. Plant Biol. 2013, 64, 839-863. [CrossRef] [PubMed]

36. Ali, S.S.; Kumar, G.B.; Khan, M.; Doohan, F.M. Brassinosteroid enhances resistance to fusarium diseases of barley. Phytopathology 2013, 103, 1260-1267. [CrossRef] [PubMed]

37. Fones, H.N.; Eyles, C.J.; Bennett, M.H.; Smith, J.A.; Preston, G.M. Uncoupling of reactive oxygen species accumulation and defence signalling in the metal hyperaccumulator plant Noccaea caerulescens. New Phytol. 2013, 199, 916-924. [CrossRef]

38. Zhang, J.; Wang, F.; Liang, F.; Zhang, Y.; Ma, L.; Wang, H.; Liu, D. Functional analysis of a pathogenesis-related thaumatin-like protein gene TaLr35PR5 from wheat induced by leaf rust fungus. BMC Plant Biol. 2018, 18, 76. [CrossRef]

39. Okada, K.; Abe, H.; Arimura, G. Jasmonates induce both defense responses and communication in monocotyledonous and dicotyledonous plants. Plant Cell Physiol. 2015, 56, 6-27. [CrossRef]

40. Frey, M.; Schullehner, K.; Dick, R.; Fiesselmann, A.; Gierl, A. Benzoxazinoid biosynthesis, a model for evolution of secondary metabolic pathways in plants. Phytochemistry 2009, 70, 1645-1651. [CrossRef]

41. Huffaker, A.; Kaplan, F.; Vaughan, M.M.; Dafoe, N.J.; Ni, X.; Rocca, J.R.; Alborn, H.T.; Teal, P.E.; Schmelz, E.A. Novel acidic sesquiterpenoids constitute a dominant class of pathogen-induced phytoalexins in maize. Plant Physiol. 2011, 156, 2082-2097. [CrossRef] [PubMed]

42. Sue, M.; Nakamura, C.; Nomura, T. Dispersed benzoxazinone gene cluster: molecular characterization and chromosomal localization of glucosyltransferase and glucosidase genes in wheat and rye. Plant Physiol. 2011, 157, 985-997. [CrossRef] [PubMed]

43. Dafoe, N.J.; Huffaker, A.; Vaughan, M.M.; Duehl, A.J.; Teal, P.E.; Schmelz, E.A. Rapidly induced chemical defenses in maize stems and their effects on short-term growth of Ostrinia nubilalis. J. Chem. Ecol. 2011, 37, 984-991. [CrossRef] [PubMed]

44. Betsiashvili, M.; Ahern, K.R.; Jander, G. Additive effects of two quantitative trait loci that confer Rhopalosiphum maidis (corn leaf aphid) resistance in maize inbred line Mo17. J. Exp. Bot. 2015, 66, 571-578. [CrossRef] [PubMed]

45. Niemeyer, H.M. Hydroxamic acids (4-hydroxy-1,4-benzoxazin-3-ones), defence chemicals in the Gramineae. Phytochemistry 1988, 27, 3349-3358. [CrossRef]

46. Rostás, M. The effects of 2,4-dihydroxy-7-methoxy-1,4-benzoxazin-3-one on two species of Spodoptera and the growth of Setosphaeria turcica in vitro. J. Pest Sci 2007, 80, 35-40. [CrossRef] 
47. Wilkes, M.A.; Marshall, D.R.; Copeland, L. Hydroxamic acids in cereal roots inhibit the growth of take-all. Soil Biol. Biochem. 1999, 31, 1831-1836. [CrossRef]

48. Van Hulten, M.; Pelser, M.; Van Loon, L.C.; Pieterse, C.M.J.; Ton, J. Costs and benefits of priming for defense in Arabidopsis. Proc. Natl. Acad.Sci. USA 2006, 103, 5602-5607. [CrossRef]

49. Conrath, U.; Beckers, G.J.M.; Flors, V.; Garcia-Agustin, P.; Jakab, G.; Mauch, F.; Newman, M.A.; Pieterse, C.M.J.; Poinssot, B.; Pozo, M.J.; et al. Priming: getting ready for battle. Mol. Plant Microbe Interact. 2006, 19, 1062-1071. [CrossRef]

50. Engelberth, J.; Alborn, H.T.; Schmelz, E.A.; Tumlinson, J.H. Airborne signals prime plants against insect herbivore attack. Proc. Natl. Acad. Sci. USA 2004, 101, 1781-1785. [CrossRef]

51. Thevenet, D.; Pastor, V.; Baccelli, I.; Balmer, A.; Vallat, A.; Neier, R.; Glauser, G.; Mauch-Mani, B. The priming molecule $\beta$-aminobutyric acid is naturally present in plants and is induced by stress. New Phytol. 2017, 213, 552-559. [CrossRef] [PubMed]

52. Ton, J.; D’Alessandro, M.; Jourdie, V.; Jakab, G.; Karlen, D.; Held, M.; Mauch-Mani, B.; Turlings, T.C. Priming by airborne signals boosts direct and indirect resistance in maize. Plant J. 2006, 49, 16-26. [CrossRef] [PubMed]

(C) 2020 by the authors. Licensee MDPI, Basel, Switzerland. This article is an open access article distributed under the terms and conditions of the Creative Commons Attribution (CC BY) license (http://creativecommons.org/licenses/by/4.0/). 